WASA Contractor Reprort 195018

TC. SW Reprom No. 94.99
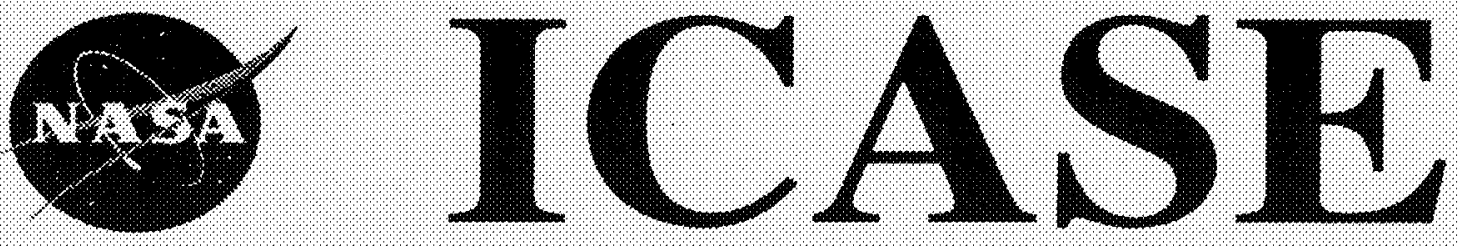

\title{
AN INVERSE PROBLEM IN THERMAL IMAGING
}

\section{Kurt Bryan}

Lester F, Caudill, Jr.

$$
\begin{aligned}
& \text { (NASA-CR-19501B) AN INVERSE } \\
& \text { PROPLEM IN THERMAL IMAGING Final } \\
& \text { RePORt (ICASE) } 35 \mathrm{p}
\end{aligned}
$$

$N 95-1347$

Unclas

$63 / 64 \quad 0034989$

Contract $)$ A S 1.9480

Wevernber 1994

Institute for Computer Applications in Sctence and Engineering NASA L angley Research Center

Mampron. VA 23681 -0001 
WASA Contractar Report 195018

TCASR, Repom No. 94,99
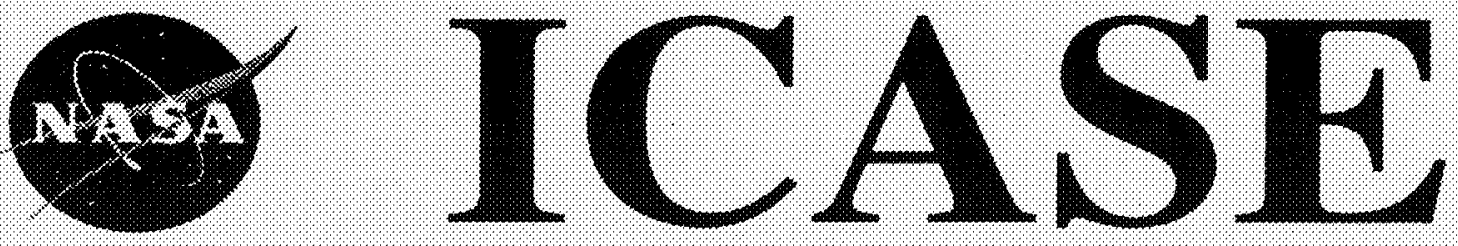

\section{AN INVERSE PROBLEM IN THERMAL IMAGING}

\section{Kurt Bryan}

Lester F, Caudill, Jr.

$$
\begin{aligned}
& \text { (NASA-CR-195018) AN INVERSE } \\
& \text { PRORLEM IN THERMAL IMAGING Final } \\
& \text { RePORt (ICASE) } 35 \mathrm{p}
\end{aligned}
$$

Conirac VAS $\}-19480$

Tecember 1904

Instiute for Computer Applications in Science and Engineering NASA Langley Research Center

Mamporan, VA 23681 -0001 


\title{
An Inverse Problem in Thermal Imaging*
}

\author{
Kurt Bryan \\ Department of Mathematics \\ Rose-Hulman Institute of Technology \\ Lester F. Caudill, Jr. \\ Department of Mathematics \\ University of Kentucky
}

\begin{abstract}
This paper examines uniqueness and stability results for an inverse problem in thermal imaging. The goal is to identify an unknown boundary of an object by applying a heat flux and measuring the induced temperature on the boundary of the sample. The problem is studied both in the case in which one has data at every point on the boundary of the region and the case in which only finitely many measurements are available. An inversion procedure is developed and used to study the stability of the inverse problem for various experimental configurations.
\end{abstract}

*This research was partially carried out while the first author was in residence at the Institute for Computer Applications in Science and Engineering (ICASE), NASA Langley Research Center, Hampton, VA 23681-0001, which is operated under National Aeronautics and Space Administration Contract No. NAS119480 . 


\section{Introduction}

Thermal imaging is a technique of wide utility in non-destructive testing and evaluation. The technique is used to recover information about the internal condition of an object by applying a heat flux to its boundary and observing the resulting temperature response on the object's surface. From this information one attempts to determine the internal thermal properties of the object, or the shape of some unknown portion of the boundary. 'Thermal imaging has been much investigated as a method for detecting damage or corrosion in aircraft. See [10] for an account of the technology and typical data processing techniques that are employed, and a more extensive bibliography on the subject.

One of the most common uses of thermal imaging is for the detection so-called "back surface" corrosion and damage. Briefly, one attempts to determine whether some inaccessible portion of an object's boundary has corroded, and therefore changed shape. In this paper we investigate the inverse problem of determining changes in the boundary profile of a twodimensional sample by using thermal imaging. We consider a certain portion of the surface of a rectangular sample to be accessible for measurements and the remainder of the surface, which may be corroded, inaccessible. This problem has been considered by others [3, 4] with an emphasis on recovering estimates of the unknown surface from data by using an output least-squares method.

We examine both a continuous and finite data version of the inverse problem. The continuous version assumes that one has data at every point on the accessible portion of the object's surface. The finite data version assumes that only finitely many measurements have been made. Our goals are

- To examine uniqueness and continuous dependence results for the continuous version of the inverse problem, and what they imply for the finite data inverse problem.

- To examine how various experimental parameters affect stability and resolution for the finite data inverse problem, especially the effect of measurement locations on stability.

- To determine how one might incorporate a priori information or assumptions into the finite data inverse problem. 
Our main focus is not to develop inversion algorithms, but in the course of examining the problem we derive (but do not prove convergence for) an inversion procedure for the finite data inverse problem. This algorithm allows the easy incorporation of a priori assumptions into the inversion process. We apply the algorithm to several simulated data sets to illustrate

our conclusions. Our study of the stability of the inverse problem reduces to studying the invertibility of a certain matrix, which we do with a singular value decomposition. We do not make any explicit finite dimensional parameterization of the unknown surface.

We should note that a very similar approach has been used in [8] to study resolution and stability for the inverse conductivity problem. Isaacson, Cheney, and others [6, 7] have also carried out similar sensitivity studies related to the inverse conductivity problem, especially the effect of finitely many measurements on the inversion process.

The outline of the paper is as follows. In Section 2 we present the mathematical formulation of the continuous and finite data versions of the inverse problem. In Section 3 we derive a linearized version of the continuous problem, and in Section 4 we show how this leads (as thermal inverse problems often do) to a first kind integral equation which must be inverted. In Section 5 we use the integral equation formulation to examine uniqueness and stability results for the linearized version of the inverse problem. In Section 6 we consider an algorithm for solving the finite data version of the inverse problem and how this approach can be used quantify the stability of the problem. In the last section we present a variety of numerical studies to examine the effects that various experimental parameters have on the stability and resolution of the inversion process, and the effect of incorporating a priori assumptions into the inversion procedure.

\section{The Inverse Problem}

Consider a sample to be imaged as a two-dimensional region $\Omega$ lying between the two surfaces $x_{2}=S\left(x_{1}\right)$ and $x_{2}=1$ as illustrated below. 


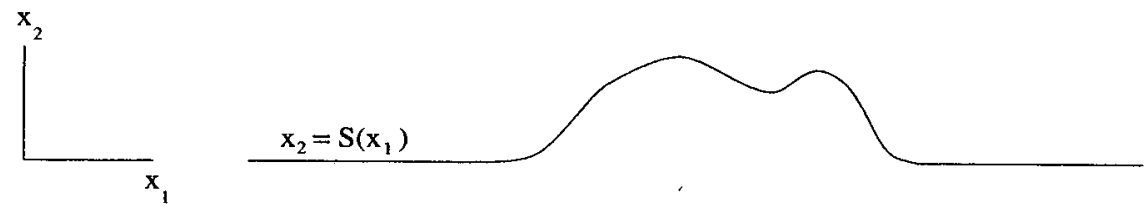

Figure 1: Sample geometry.

We will refer to the surface $x_{2}=1$ as the "top" or "front" surface and $x_{2}=S\left(x_{1}\right)$ as the "back" surface. We assume that the ends of the sample are sufficiently far away that they can be ignored, so for our purposes the sample is unbounded in the $x_{1}$ direction. The top surface is assumed to be accessible for inspection or measurements, but the back surface $x_{2}=S\left(x_{1}\right)$ is inaccessible. This is the portion of the sample to be inspected for corrosion. The ideal uncorroded case is a flat back surface $S\left(x_{1}\right) \equiv 0$. In the corroded case above $S\left(x_{1}\right)>0$ for some values of $x_{1}$. We will assume that the function $S$ belongs to $H^{2}(\mathbb{R})$, although this assumption will later be relaxed. In particular, since $H^{2}(\mathbb{R}) \subset C^{1}(\mathbb{R})$ there is a continuous unit normal vector field on the back surface. The goal is to determine the back surface or the function $S$ by taking measurements only on the front surface.

A time-dependent heat flux $g\left(x_{1}, t\right)$ is applied to the top of the sample $x_{2}=1$. We will assume that the sample material is homogeneous with thermal diffusivity $\kappa$ and thermal conductivity $\alpha$, both known constants. We will use $T(x, t)$ to denote the resulting temperature induced in $\Omega$, where $x=\left(x_{1}, x_{2}\right)$. The direct thermal diffusion problem will be modeled by the standard heat equation

$$
\begin{aligned}
\frac{\partial T}{\partial t}-\kappa \Delta T & =0 \text { in } \Omega \\
\alpha \frac{\partial T}{\partial \nu} & =g\left(x_{1}, t\right) \text { on } x_{2}=1, \\
\alpha \frac{\partial T}{\partial \nu} & =0 \text { on } x_{2}=S\left(x_{1}\right), \\
T(x, 0) & =T_{0}(x)
\end{aligned}
$$

for $t>0$, where $\frac{\partial}{\partial \nu}$ denotes the outward normal derivative on the boundary of $\Omega$. The function $T_{0}(x)$ is the initial temperature of the region $\Omega$ at time $t=0$. Note that the back surface is assumed to block all heat conduction. 
We will also assume that the heat flux $g\left(x_{1}, t\right)$ is periodic, of the form $\operatorname{Re}\left[g\left(x_{1}\right) e^{i \omega t}\right]$ with $\omega>0$. For simplicity, we also take the constants $\kappa$ and $\alpha$ equal to one. Under these assumptions the solution to equation (2.1) is given as $T(x, t)=\operatorname{Re}\left[e^{i \omega t} u(x)\right]$ where $u(x)$ satisfies

$$
\begin{aligned}
\Delta u-i \omega u & =0 \text { in } \Omega \\
\frac{\partial u}{\partial \nu} & =g\left(x_{1}\right) \text { on } x_{2}=1 \\
\frac{\partial u}{\partial \nu} & =0 \text { on } x_{2}=S\left(x_{1}\right)
\end{aligned}
$$

at least after transients from the initial condition have died out. The main case of interest is that in which $g\left(x_{1}\right)$ is constant, corresponding to uniform heating of the outer surface. This is typically the case when heat or flash lamps are used to provide the input flux $g$. For the moment, however, we will not restrict $g$.

There are two versions of the inverse problem to be considered:

\section{Continuous Version:}

Given measurements of $u(x)$ at all points on the top surface $x_{2}=1$, determine the function $S\left(x_{1}\right)$.

\section{Finite Data Version:}

Given measurements of $u(x)$ on the top surface $x_{2}=1$ at points $x_{1}=a_{1}, a_{2}, \ldots, a_{n}$, estimate the function $S\left(x_{1}\right)$.

The finite data version corresponds to the case in which one has actual measurements. The data need not be actual point measurements of the temperature $u$, but this is the most common situation. Of particular interest are the questions

1. Can the function $S\left(x_{1}\right)$ be uniquely determined by knowing $u\left(x_{1}\right)$ for all $x_{1}$ on the top surface?

2. If $S\left(x_{1}\right)$ is uniquely determined by $u\left(x_{1}\right)$, how sensitive is $S\left(x_{1}\right)$ to perturbations in the data? What kinds of features in the back surface $x_{2}=S\left(x_{1}\right)$ can or cannot be easily determined from the data? 
3. Since any practical application falls under the finite data formulation, how stable is the estimate of $S\left(x_{1}\right)$ based on finitely many pieces of data? What factors influence stability in this case, and is there an inversion procedure to produce a reasonable estimate of $S\left(x_{1}\right)$ using finitely many measurements?

The first question is easily answered "yes" by a standard argument. This is the content of the following-result.

Theorem 2.1 (Uniqueness) Let $u\left(x_{1}, x_{2} ; S\right)$ denote the solution to (2.2) with back surface $S$ and nonzero flux $g$. If $u\left(x_{1}, 1 ; S_{1}\right)=u\left(x_{1}, 1 ; S_{2}\right)$ for each $\left(x_{1}, 1\right)$ in an open subset $C$ of the top surface of $\Omega$, then $S_{1}=S_{2}$.

Proof: Suppose $S_{1} \neq S_{2}$. Using the shorthand notation $u_{i} \equiv u\left(x_{1}, x_{2} ; S_{i}\right)$, we have that $u_{1}$ and $u_{2}$ have the same Cauchy data on $C$, and by unique continuation $u_{1}$ and $u_{2}$ agree on any connected domain on which both are defined, provided that that domain contains an open portion of $C$. Assume that $S_{1}$ and $S_{2}$ are not equal and denote by $\Omega_{i}$ the region bounded by $x_{2}=1$ and $x_{2}=S_{i}\left(x_{1}\right) . S_{1} \neq S_{2}$, so there is some non-empty connected component $D$ of $\Omega_{1} \backslash \Omega_{2}$ or $\Omega_{2} \backslash \Omega_{1}$. Let us suppose the latter so that the region $D$ is bounded by $x_{2}=S_{1}\left(x_{1}\right)$ and $x_{2}=S_{2}\left(x_{1}\right)$. On $x_{2}=S_{2}\left(x_{1}\right)$ we know that the normal derivative of $u_{2}$ is identically zero; on $x_{2}=S_{1}\left(x_{1}\right)$ we know that the normal derivative of $u_{1}$ is zero (from inside $\Omega_{1}$ ) and since $u_{2} \equiv u_{1}$ and $u_{2}$ is smooth across $x_{2}=S_{1}\left(x_{1}\right)$, we conclude that the normal derivative of $u_{2}$ vanishes on the boundary of $D$. This forces $u_{2} \equiv 0$ inside $D$. Standard elliptic regularity arguments force $u_{2} \equiv 0$ inside $\Omega_{2}$, which implies that the flux $g$ is identically zero, a contradiction. Thus we must have $S_{1} \equiv S_{2}$ and so the back surface $S\left(x_{1}\right)$ is uniquely determined by the boundary data on any open portion of the top surface.

The second and third questions will be examined in the next few sections by considering a linearization of the original problem.

\section{A Linearization}

We now linearize the original direct problem given by equation (2.2) with respect to the function $S$. Let $u_{0}(x)$ denote the solution to $(2.2)$ with $S \equiv 0$. The surface $x_{2} \equiv 0$ is the 
point at which we will linearize, since this represents the uncorroded or ideal profile from which we hope to detect any deviation.

Let $\Omega_{0}$ denote the region $\left\{\left(x_{1}, x_{2}\right): 0<x_{2}<1\right\}$ in $\mathbb{R}^{2}$. For any $S \in H^{2}(\mathbb{R}) \subset C^{1}(\mathbb{R})$, let us construct the map $\phi$ from $\Omega$ to $\Omega_{0}$ by

$$
\phi\left(x_{1}, x_{2}\right)=\left(x_{1}, \frac{x_{2}-S\left(x_{1}\right)}{1-S\left(x_{1}\right)}\right) .
$$

If $\left|S\left(x_{1}\right)\right|<1$ then it is easy to check that $\phi$ is invertible on $\Omega_{0}$. This map fixes the top boundary of $\Omega$ and maps the bottom surface to $x_{2}=0$. Let $y=\phi(x)$ and $v(y)=u\left(\phi^{-1}(y)\right)=$ $u(x)$. Under such a change of coordinates $\nabla_{x}=(D \phi)^{T} \nabla_{y}$ and $\frac{\partial}{\partial \nu_{x}}=\frac{\partial}{\partial \eta_{y}}$ where $\eta_{y}=(D \phi) \nu_{x}$, $(D \phi)$ is the derivative of $\phi$, and $(D \phi)^{T}$ is the transpose. Under this change of coordinates the boundary value problem (2.2) becomes

$$
\begin{aligned}
\nabla \cdot \kappa \nabla v-i \omega v & =0 \text { in } \Omega_{0} \\
\frac{\partial v}{\partial \eta} & =g\left(x_{1}\right) \text { on } x_{2}=1, \\
\frac{\partial v}{\partial \eta} & =0 \text { on } x_{2}=0
\end{aligned}
$$

where $\kappa=(D \phi)(D \phi)^{T}$. The vector $\eta$ can be written as

$$
\eta=\left[\begin{array}{c}
0 \\
\frac{1}{1-S\left(x_{1}\right)}
\end{array}\right] \text { on } x_{2}=1, \quad \eta=\frac{1}{\sqrt{1+\left(S^{\prime}\left(x_{1}\right)\right)^{2}}}\left[\begin{array}{c}
S^{\prime}\left(x_{1}\right) \\
\frac{1+\left(S^{\prime}\left(x_{1}\right)\right)^{2}}{S\left(x_{1}\right)-1}
\end{array}\right] \text { on } x_{2}=0
$$

This change of coordinates removes the unknown $S$ from the definition of the boundary of $\Omega$ and puts $S$ into the coefficients of the heat operator and boundary conditions.

Now we linearize the problem with respect to $S$ about $S \equiv 0$ by assuming that $S=\epsilon \tilde{S}$ for some function $\tilde{S}$, where $\epsilon$ is some small real number. Let $u$ denote the solution to (3.3) for a general $S$ and $u_{0}$ the solution to equation (3.3) for $S \equiv 0$. Suppose that the resulting perturbation in $u_{0}$ can be represented as $u=u_{0}+\epsilon v_{0}$. If we substitute these relations into (3.3), use the fact that $u_{0}$ satisfies (3.3) with $S=0$, and drop all quadratic $\epsilon$ terms then we obtain a linearized version of the direct problem,

$$
\begin{aligned}
\Delta v_{0}-i \omega v_{0} & =-\nabla \cdot\left[\tilde{\kappa} \nabla u_{0}\right], \text { in } \Omega_{0} \\
\frac{\partial v_{0}}{\partial \nu} & =-\tilde{S}\left(y_{1}\right) g\left(y_{1}\right), \text { on } y_{2}=1 \\
\frac{\partial v_{0}}{\partial \nu} & =-\tilde{S}^{\prime}\left(y_{1}\right) u_{0}^{\prime}\left(y_{1}\right), \text { on } y_{2}=0
\end{aligned}
$$


where $\tilde{\kappa}(y)$ is the matrix

$$
\tilde{\kappa}(y)=\left[\begin{array}{cc}
0 & \left(y_{2}-1\right) \tilde{S}^{\prime}\left(y_{1}\right) \\
\left(y_{2}-1\right) \tilde{S}^{\prime}\left(y_{1}\right) & 2 \tilde{S}\left(y_{1}\right)
\end{array}\right] .
$$

In particular, we are interested in the special case $g \equiv 1$ corresponding to spatially uniform periodic heating of the top surface. Under this condition the function $u_{0}$ depends only on $x_{2}$ and equation (3.3) for $u_{0}$ becomes a two-point boundary value problem

$$
\begin{aligned}
u_{0}^{\prime \prime}-i \omega u_{0} & =0 \text { on }(0,1), \\
u_{0}^{\prime}(0) & =0 \\
u_{0}^{\prime}(1) & =1 .
\end{aligned}
$$

If we now use $u$ rather than $v_{0}$ to denote the temperature perturbation satisfying equation (3.3) then the linearized version of the problem for $g \equiv 1$ is then

$$
\begin{aligned}
\Delta u-i \omega u & =\left(1-x_{2}\right) u_{0}^{\prime}\left(x_{2}\right) S^{\prime \prime}\left(x_{1}\right)-2 u_{0}^{\prime \prime}\left(x_{1}\right) S\left(x_{1}\right) \text { on } \Omega_{0}, \\
\frac{\partial u}{\partial \nu} & =-S\left(x_{1}\right) \text { on } x_{2}=1, \\
\frac{\partial u}{\partial \nu} & =0 \text { on } x_{2}=0 .
\end{aligned}
$$

For simplicity, this is the version of the problem we will examine, although the more general linearized version (3.4) can be examined using similar techniques. Note that the linearized problem is defined on the domain $\Omega_{0}$ which does not depend on $S$.

\section{An Integral Identity}

Let the function $d(a)=u(a, 1)$ denote the top surface data from the direct problem (3.6). Given that the relation between $S$ and $d$ is linear, it seems reasonable that this relationship can be expressed by an integral operator

$$
d(a)=\int_{-\infty}^{\infty} \phi_{a}(y) S(y) d y
$$

where $\phi_{a}$ is some function which depends on the particular point $a$. Such a relationship does exist and we can say quite a bit about the function(s) $\phi_{a}$, as we now demonstrate. 
Let $\mathrm{L}=\Delta-i \omega$. By Green's second identity

$$
\int_{\Omega_{0}}(\phi \mathrm{L} u-u \mathrm{~L} \phi) d x=\int_{x_{2}=1}\left(\phi \frac{\partial u}{\partial \nu}-u \frac{\partial \phi}{\partial \nu}\right) d x_{1}+\int_{x_{2}=0}\left(\phi \frac{\partial u}{\partial \nu}-u \frac{\partial \phi}{\partial \nu}\right) d x_{1}
$$

where $\phi$ is any sufficiently regular function defined on $\Omega$. Assume that $u$ is a solution to (3.6) and that $\phi$ is a function which satisfies $L \phi=0$ on $\Omega_{0}$ and $\frac{\partial \phi}{\partial \nu}=0$ on the surface $x_{2}=0$. Then the above equation becomes

$$
\begin{aligned}
\int_{\Omega_{0}} \phi(x)\left(\left(1-x_{2}\right) u_{0}^{\prime}\left(x_{2}\right) S^{\prime \prime}\left(x_{1}\right)-2 u_{0}^{\prime \prime}\left(x_{1}\right) S^{\prime}\left(x_{1}\right)\right) d x & +\int_{-\infty}^{\infty} \phi\left(x_{1}, 1\right) S\left(x_{1}\right) d x_{1} \\
& =-\int_{-\infty}^{\infty} \frac{\partial \phi}{\partial \nu}\left(x_{1}, 1\right) d\left(x_{1}\right) d x_{1}
\end{aligned}
$$

Let us now complete the specification of $\phi$ by requiring $\frac{\partial \phi}{\partial \nu}=\delta_{a}$ on the top surface, where $\delta_{a}$ denotes a delta function on the surface $x_{2}=1$ at the point $x_{1}=a$. If we write $\phi_{a}$ to denote the dependence of $\phi$ on $a$ then we obtain

$$
\int_{\Omega_{0}} \phi_{a}(x)\left(\left(1-x_{2}\right) u_{0}^{\prime}\left(x_{2}\right) S^{\prime \prime}\left(x_{1}\right)-2 u_{0}^{\prime \prime}\left(x_{1}\right) S\left(x_{1}\right)\right) d x+\int_{-\infty}^{\infty} \phi_{a}\left(x_{1}, 1\right) S\left(x_{1}\right) d x_{1}=-d(a) .
$$

Note that this equation involves no unknown quantities except $S$ on the left side.

It is worth saying a few words about the function $\phi_{\boldsymbol{a}}$ which satisfies

$$
\begin{aligned}
\Delta \phi_{a}-i \omega \phi_{a} & =0 \text { in } \Omega_{0}, \\
\frac{\partial \phi_{a}}{\partial \nu} & =\delta_{a} \text { on } x_{2}=1 \\
\frac{\partial \phi_{a}}{\partial \nu} & =0 \text { on } x_{2}=0
\end{aligned}
$$

Let $\Gamma(x)$ be a Green's function for the operator $\Delta-i \omega$ on $\mathbb{R}^{2}$; such a function is given by

$$
\Gamma(x)=\frac{-1}{2 \pi}(\operatorname{ker}(r \sqrt{w})+i \operatorname{kei}(r \sqrt{w}))
$$

where $r=|x|=\sqrt{x_{1}^{2}+x_{2}^{2}}$ and $\operatorname{ker}()$ and $\operatorname{kei}()$ are the Kelvin functions (see [1], section 9.9). The function $\operatorname{ker}(r)$ has a $-\ln (r)$ singularity as $r$ approaches zero, while kei $(r)$ is bounded. Both functions and their derivatives are smooth away from zero and rapidly decreasing as $r$ tends to infinity, where "rapidly decreasing" means faster than any power of $\frac{1}{r}$. If we define $\psi_{a}(x)=-2 \Gamma\left(x-x_{a}\right)$ where $x_{a}$ is the point $(a, 1)$ on the top surface, then standard potential theory arguments ([9], chapter 3$)$ show that

$$
\begin{aligned}
\Delta \psi_{a}-i \omega \psi_{a} & =0 \text { in } \Omega_{0} \\
\frac{\partial \psi_{a}}{\partial \nu} & =\delta_{a} \text { on } x_{2}=1
\end{aligned}
$$


It is not true that

$$
\frac{\partial \psi_{a}}{\partial \nu}=0 \text { on } x_{2}=0 .
$$

However, if we take $v_{a} \in H^{1}(\Omega)$ to satisfy

$$
\begin{aligned}
\Delta v_{a}-i \omega v_{a} & =0 \text { in } \Omega_{0} \\
\frac{\partial v_{a}}{\partial \nu} & =0 \text { on } x_{2}=1 \\
\frac{\partial v_{a}}{\partial \nu} & =-\frac{\partial \psi_{a}}{\partial \nu} \text { on } x_{2}=0
\end{aligned}
$$

then $\phi_{a}=\psi_{a}+v_{a}$. Since the Neumann data for $v_{a}$ on the bottom surface is $-\frac{\partial \psi_{a}}{\partial \nu}$ which is in $H^{\infty}(\mathbb{R})$ (the singularity for $\psi_{a}$ lies on the top surface, and away from this singularity $\psi_{a}$ is smooth and rapidly decreasing), one can show that $v_{a}$ is in $H^{\infty}\left(\Omega_{0}\right)$. As a result, the function $\phi_{a}(x)$ has a $-\frac{1}{\pi} \ln |x|$ singularity near $x=0$ and otherwise is smooth and rapidly decreasing in $|x|$, along with its derivatives of all orders.

If we write the integrals in equation (4.7) with limits, we find that $S$ must satisfy

$$
\int_{-\infty}^{\infty}\left(p_{a}\left(x_{1}\right) S^{\prime \prime}\left(x_{1}\right)+q_{a}\left(x_{1}\right) S\left(x_{1}\right)\right) d x_{1}=-d(a)
$$

where

$$
\begin{aligned}
& p_{a}\left(x_{1}\right)=\int_{0}^{1} \phi_{a}\left(x_{1}, x_{2}\right) u_{0}^{\prime}\left(x_{2}\right)\left(1-x_{2}\right) d x_{2} \\
& q_{a}\left(x_{1}\right)=-2 \int_{0}^{1} \phi_{a}\left(x_{1}, x_{2}\right) u_{0}^{\prime \prime}\left(x_{2}\right) d x_{2}+\phi_{a}\left(x_{1}, 1\right) .
\end{aligned}
$$

One can check that the integral in $q_{a}\left(x_{1}\right)$ is continuous as a function of $x_{1}$, smooth away from $x_{1}=a$, and rapidly decreasing in $x_{1}$. Also, since $\phi_{a}\left(x_{1}, 1\right)$ has a logarithmic singularity at $x_{1}=a$, so does $q_{a}$. Moreover $q_{a}$ is an $L^{2}$ function. The function $p_{a}\left(x_{1}\right)$ is also clearly smooth away from $x_{1}=a$ and rapidly decreasing in $x_{1}$. The singularity of $\phi_{a}\left(x_{1}, x_{2}\right)$ looks like the singularity of $\operatorname{ker}\left|x-x_{a}\right|$, and one can use this fact to expand the function $\phi_{\boldsymbol{a}}\left(x_{1}, x_{2}\right)$ near the singularity to show that the function $p_{a}\left(x_{1}\right)$ is actually in $H^{2}(\mathbb{R})$. Since both $p_{a}\left(x_{1}\right)$ and $q_{a}\left(x_{1}\right)$ tend rapidly to zero as $\left|x_{1}\right| \rightarrow \infty$ we can integrate by parts twice to find that

$$
\int_{-\infty}^{\infty} p_{a}\left(x_{1}\right) S^{\prime \prime}\left(x_{1}\right) d x_{1}=\int_{-\infty}^{\infty} p_{a}^{\prime \prime}\left(x_{1}\right) S\left(x_{1}\right) d x_{1}
$$

Equation (4.9) can now be written

$$
\int_{-\infty}^{\infty} c_{a}\left(x_{1}\right) S\left(x_{1}\right) d x_{1}=d(a)
$$


where

$$
c_{a}\left(x_{1}\right)=-p_{a}^{\prime \prime}\left(x_{1}\right)-q_{a}\left(x_{1}\right) \in L^{2}(\mathbb{R})
$$

Given the translation invariance of this problem in the $x_{1}$ direction and the fact that the flux $g\left(x_{1}\right) \equiv 1$ does not depend on $x_{1}$, it is clear that $c_{a}\left(x_{1}\right)=c\left(x_{1}-a\right)$ for some function $c$.

Lemma 4.1 The function $c\left(x_{1}\right)=u_{0}(0) i \omega \phi_{0}\left(x_{1}, 0\right)$, where $\phi_{0}$ satisfies (4.8) with $a=0$ and $u_{0}$ satisfies (3.5).

In proving this result, We will make use of the following simple fact.

Remark 4.1 If $f(t)$ and $g(t)$ are functions defined on $[0,1]$ with $f^{\prime}(0)=f^{\prime}(1)=0$ and $g^{\prime}(0)=0, g^{\prime}(1)=1$, and $g^{\prime \prime}=i \omega g$ then

$$
\int_{0}^{1} f^{\prime \prime}(t) g^{\prime}(t)(1-t) d t=f(1)-2 i \omega \int_{0}^{1} f(t) g(t) d t+i \omega f(0) g(0)+i \omega \int_{0}^{1}(1-t) f(t) g^{\prime}(t) d t
$$

To see this identity requires only a few applications of integration by parts, $\int u d v=u v-$ $\int v d u$. Take $u=g^{\prime}(t)(1-t), d v=f^{\prime \prime}(t) d t$ to obtain

$$
\int_{0}^{1} f^{\prime \prime}(t) g^{\prime}(t)(1-t) d t=\int_{0}^{1} f^{\prime}(t) g^{\prime}(t) d t-i \omega \int_{0}^{1} f^{\prime}(t) g(t)(1-t) d t
$$

where we have made use of $g^{\prime \prime}=i \omega g$. Integrate the first integral by parts with $u=g^{\prime}$ and $d v=f^{\prime} d t$; integrate the second integral by parts with $u=(1-t) g$ and $d v=f^{\prime} d t$. After a number of cancellations, the Remark follows immediately.

Proof of Lemma 4.1: From equations (4.11), (4.12) and (4.14) we obtain

$$
c\left(x_{1}\right)=-\int_{0}^{1}\left(\left(1-x_{2}\right) \frac{\partial^{2} \phi_{0}}{\partial x_{1}^{2}} u_{0}^{\prime}\left(x_{2}\right)-2 \phi_{0} u_{0}^{\prime \prime}\left(x_{1}\right)\right) d x_{2}-\phi_{0}\left(x_{1}, 1\right)
$$

Recall that $u_{0}$ is a function of $x_{2}$ only, and that $u_{0}^{\prime \prime}=i \omega u_{0}$ with $u_{0}^{\prime}(0)=0$ and $u_{0}^{\prime}(1)=1$. Since $\Delta \phi_{0}-i \omega \phi_{0}=0$ we have $\frac{\partial^{2} \phi_{0}}{\partial x_{1}^{2}}=i \omega \phi_{0}-\frac{\partial^{2} \phi_{0}}{\partial x_{1}^{2}}$. With this substitution and $u_{0}^{\prime \prime}=i \omega u_{0}$

$$
c\left(x_{1}\right)=-\int_{0}^{1}\left[i \omega\left(1-x_{2}\right) \phi_{0} u_{0}^{\prime}\left(x_{2}\right)-\left(1-x_{2}\right) u_{0}^{\prime} \frac{\partial^{2} \phi_{0}}{\partial x_{2}^{2}}-2 i \omega \phi_{0} u_{0}\right] d x_{2}-\phi_{0}\left(x_{1}, 1\right) .
$$

To finish the proof, use Remark 4.1 above with $f=\phi_{0}$ and $g=u_{0}$. After a few cancellations the statement of the lemma follows. 
Lemma 4.1 implies a number of things about the function $c\left(x_{1}\right)$, in particular, that $c$ is in $H^{\infty}(\mathbb{R})$. It is important to note that $u_{0}(0)$ is never zero, for $u_{0}$ satisfies the two-point boundary value problem (3.5) and one easily finds that

$$
u_{0}\left(x_{2}\right)=\frac{e^{\alpha x_{2}}+e^{-\alpha x_{2}}}{\alpha\left(e^{\alpha}-e^{-\alpha}\right)},
$$

where $\alpha=(1+i) \sqrt{\omega / 2}$. In particular,

$$
u_{0}(0)=\frac{2}{\alpha\left(e^{\alpha}-e^{-\alpha}\right)} .
$$

The numerator above is never zero, hence $u_{0}(0) \neq 0$. As a result the function $c\left(x_{1}\right)$ will not be identically zero for any $\omega>0$.

There is one more fact which will be extremely useful. Defining the Fourier transform $\hat{f}(y)$ of a function $f(x)$ of a single variable by

$$
\hat{f}(y)=\int_{-\infty}^{\infty} e^{-i x y} f(x) d x
$$

we have

Lemma 4.2 For c given by Lemma 4.1,

$$
\hat{c}(y)=\frac{2 i \omega \iota_{0}(0)}{\alpha\left(e^{\alpha}-e^{-\alpha}\right)}
$$

where $\alpha=\sqrt{y^{2}+i \omega}$. Also, the function $\hat{c}(y)$ is never equal to zero.

Proof: Let $\hat{\phi}_{0}\left(y_{1}, x_{2}\right)$ denote the Fourier transform of $\phi_{0}\left(x_{1}, x_{2}\right)$ with respect to $x_{1}$ only, where $\phi_{0}$ satisfies equation (4.8) with $a=0$. Then

$$
\hat{\phi}_{0}\left(y_{1}, x_{2}\right)=\int_{-\infty}^{\infty} \phi_{0}\left(x_{1}, x_{2}\right) e^{-i x_{1} y_{1}} d x_{1} .
$$

If $\phi_{0}$ is sufficiently rapidly decreasing (as in our case) then $\frac{\partial}{\partial x_{2}}$ and the Fourier transform operator commute. Fourier transforming both sides of the boundary value problem (4.8) with respect to $x_{1}$ yields a two-point boundary value problem for $\hat{\phi}_{0}\left(y_{1}, x_{2}\right)$ in the $x_{2}$ variable,

$$
\begin{aligned}
\frac{d^{2} \hat{\phi}_{0}}{d x_{2}^{2}}-\left(y_{1}^{2}+i \omega\right) \hat{\phi}_{0} & =0 \\
\frac{d \hat{\phi}_{0}}{d x_{2}}(1) & =1, \\
\frac{d \hat{\phi}_{0}}{d x_{2}}(0) & =0
\end{aligned}
$$


where we have used $\hat{\delta}_{0}=1$. The solution is

$$
\hat{\phi}_{0}\left(y_{1}, x_{2}\right)=\frac{e^{\alpha x_{2}}+e^{-\alpha x_{2}}}{\alpha\left(e^{\alpha}-e^{-\alpha}\right)}
$$

where $\alpha=\sqrt{y_{1}^{2}+i \omega}$. On the bottom surface $x_{2}=0$

$$
\hat{\phi}_{0}\left(y_{1}, 0\right)=\frac{2}{\alpha\left(e^{\alpha}-e^{-\alpha}\right)}
$$

which, combined with Lemma 4.1 yields the conclusion. The fact that $\hat{c}(y)$ is never zero follows from $\omega>0$ and $u_{0}(0) \neq 0$.

\section{The Linearized Inverse Problem}

In this section we will examine the continuous version of the linearized inverse problem. Suppose that we have top surface data $d\left(x_{1}\right)=u\left(x_{1}, 1\right)$ where $u$ satisfies equation (3.6). Based on equation (4.13) and the above noted fact that $c_{a}\left(x_{1}\right)=c\left(x_{1}-a\right)$ we conclude that $S$ and $d$ satisfy the relationship

$$
\int_{-\infty}^{\infty} S\left(y_{1}\right) c\left(y_{1}-x_{1}\right) d y_{1}=d\left(x_{1}\right)
$$

or

$$
\int_{-\infty}^{\infty} S\left(y_{1}\right) \phi\left(x_{1}-y_{1}\right) d y_{1}=S * \phi=d\left(x_{1}\right)
$$

where $\phi\left(x_{1}\right)=c\left(-x_{1}\right)$ and "*" denotes convolution. With $d\left(x_{1}\right)$ considered known this becomes a first kind integral equation for the unknown function $S$. The kernel $\phi$ is known, or can be determined, according to Lemma 4.1. First kind integral equations have been extensively studied $([11,12])$ and are well-known to be unstable; small perturbations in the right hand side $d(x)$ can lead to arbitrarily large changes in the solution $S$. However this formulation of the inverse problem as an integral equation will allow us to obtain uniqueness and stability estimates for the linearized version of the inverse problem.

Theorem 5.1 (Uniqueness) If the data $d(x)$ is an $L^{2}$ function and if there exists a solution $S \in L^{2}(\mathbb{R})$ to the linearized inverse problem, then $S$ is unique. 
Proof: Suppose $S_{1}$ and $S_{2}$ are $L^{2}$ functions which both give rise to data $d$. Let $S=S_{1}-S_{2}$. Linearity implies that the data for $S$ is identically zero. Fourier transform both sides of the equation $S * \phi=0$. By the convolution theorem and the fact that $\hat{\phi}=\overline{\hat{c}}$ if $\phi(x)=c(-x)$ (where the overbar denotes complex conjugation) we obtain $\hat{\phi} \hat{S}=0$. By Lemma $4.2 \hat{\phi}=$ $\overline{\hat{c}} \neq 0$ and we conclude that

$$
\hat{S}=0
$$

so that $S \equiv 0$ or $S_{1}=S_{2}$.

Remark 5.1 In the preceding proof, we assumed a priori that $S$ is in $L^{2}(\mathbb{R})$. In general, for an arbitrary $d \in L^{2}(\mathbb{R})$ we cannot find a function $S$ in $L^{2}$ which gives rise to data $d$ via equation (5.16).

The convolution equation (5.16) also provides information on continuous dependence. Since the function $\hat{\phi}_{0}$ is smooth and never equal to zero, we can define the space of functions $L_{*}^{2}(\mathbb{R})$ with the norm

$$
\|f\|_{*}^{2}=\int_{-\infty}^{\infty}\left|\frac{\hat{f}(z)}{\hat{\phi}_{0}(z)}\right|^{2} d z .
$$

From Lemma 4.2 it follows that $\frac{1}{\hat{\phi}_{0}(z)}$ grows like $z e^{z}$. The norm \|\|$_{*}$ thus puts a heavy penalty on high frequencies; the functions in this space are very smooth. We can Fourier transform both sides of equation (5.16), divide by $\hat{\phi}_{0}$ and take the $L^{2}$ norms of both sides to obtain

Theorem 5.2 (Continuous Dependence) If a back surface $x_{2}=S\left(x_{1}\right)$ generates front surface data $d(x)$ for the linearized problem (3.6), then

$$
\|S\|_{L^{2}} \leq C\|d\|_{*}
$$

where $C$ is independent of $d$.

Estimates of $S$ from data $d$ will thus be extremely sensitive to any noise, because the inversion process weights a frequency $f$ in the data by a factor proportional to $f e^{f}$. Lemma 4.2 and the structure of the convolution operator mapping $S$ to the data $d$ make it clear that it will be difficult to estimate the high spatial frequency components in the Fourier decomposition of $S$, for these components are heavily damped out by the forward mapping. 


\section{The Case of Finitely Many Measurements}

Suppose that we have point estimates $d\left(a_{i}\right)=u\left(a_{i}, 1\right)$ of the temperature on the top surface at $n$ distinct points. How can we construct a reasonable estimate of the function $S\left(x_{1}\right)$ ? How can we quantify the stability of the reconstruction with respect to errors in the data, and how does the choice of measurement locations $a_{i}$ affect the stability? Let us assume that we seek an estimate $S \in L^{2}(\mathbb{R})$. Physical considerations make it desirable to obtain an estimate with more regularity, but this will be a consequence of the proposed reconstruction procedure. Based on the convolution equation (5.16) we know that $S$ must satisfy the $n$ constraints

$$
<S, c_{i}>=\int_{-\infty}^{\infty} S\left(x_{1}\right) \bar{c}_{i}\left(x_{1}\right) d x_{1}=d\left(a_{i}\right), \quad i=1, \ldots, n,
$$

with $c_{i}\left(x_{1}\right) \equiv \bar{c}\left(a_{i}-x_{1}\right)$ where $c\left(x_{1}\right)$ is the function from Lemma 4.1 and $\left.<f, g\right\rangle=\int_{\mathbb{R}} f \bar{g}$ is the usual $L^{2}$ inner product. Note that since $c_{i}$ is an $L^{2}$ function, $S \mapsto<S, c_{i}>$ is a bounded linear functional on $L^{2}$. The set $(6.17)$ is a horribly underdetermined set of equations. We can expect to find an entire translated subspace of functions of codimension $n$ in $L^{2}(\mathbb{R})$ which satisfy the given conditions, and any such function "solves" the inverse problem, in the sense that it gives rise to the measured data.

One practical method for specifying a unique function in $L^{2}$ which solves the inverse problem is to seek that element in $L^{2}$ which satisfies the given conditions and has minimal norm. That such an element exists follows from the fact that the relations (6.17) define a closed convex subset of $L^{2}$ and hence this subset has a unique element of minimal norm. This idea has been used before ([8]) to construct a "pseudo-inverse" for the finite measurement case and to characterize the stability and information content for the inverse conductivity problem, and has also been used for reconstruction from partial information in tomographic problems [5]. The approach has several merits: In the present case it leads to an exceptionally easy and efficient inversion algorithm which allows us to study the conditioning of the inverse problem independent of any explicit finite dimensional parameterization of the unknown $S$. By weighting the $L^{2}$ space appropriately we can also incorporate a priori assumptions into the reconstruction procedure and examine the effect these assumptions have on stability. Also, given the continuous dependence result from Lemma 5.2 and the fact that data is 
invariably noisy, we know that any inversion procedure will tend to give extraneous high frequency components in any estimate of $S$; choosing the estimate of minimal norm should help to damp out spurious components in the estimate. In this sense the procedure may be viewed as a form of regularization.

It is an easy application of Lagrange multipliers to verify that the unique element of $L^{2}$ with minimum norm which satisfies the constraints (6.17) must be of the form

$$
S\left(x_{1}\right)=\sum_{k=1}^{n} \lambda_{k} c_{k}\left(x_{1}\right)
$$

for some $\left\{\lambda_{k}\right\}_{k=1}^{n} \subseteq \mathbb{C}$. The constants $\lambda_{k}$ can be determined by substituting (6.18) into equations (6.17) and solving the resulting $n \times n$ system. The system is of the form $M \lambda=d$. where $M=\left[m_{i j}\right]$ is an $n$ by $n$ matrix, $\lambda$ is the $n$ vector $\left(\lambda_{1}, \ldots, \lambda_{n}\right)^{T}$ and $d$ is an $n$ vector $\left(d\left(a_{1}\right), \ldots, d\left(a_{n}\right)\right)^{T}$. The entries of $M$ are given by

$$
m_{i j}=\int_{-\infty}^{\infty} c_{i}\left(x_{1}\right) \bar{c}_{j}\left(x_{1}\right) d x_{1}=\int_{-\infty}^{\infty} \bar{c}\left(x_{1}-a_{i}\right) c\left(x_{1}-a_{j}\right) d x_{1} .
$$

The matrix $M$ is clearly Hermitian and in fact is always invertible. To see this, suppose we can find some vector $\lambda \neq 0$ with $M \lambda=0$. Then $\bar{\lambda}^{T} M \lambda=0$ and we conclude that

$$
\int_{-\infty}^{\infty} \sum_{i, j} c\left(x_{1}-a_{i}\right) \lambda_{i} \bar{c}\left(x_{1}-a_{j}\right) \bar{\lambda}_{j} d x_{1}=\int_{-\infty}^{\infty}\left|\sum_{i=1}^{n} \lambda_{i} c\left(x_{1}-a_{i}\right)\right|^{2} d x_{1}=0 .
$$

This implies

$$
\sum_{j=1}^{n} \lambda_{j} c\left(x_{1}-a_{j}\right) \equiv 0
$$

Fourier transform both sides and use the basic properties of the Fourier transform to obtain

$$
\hat{c}(y) \sum_{j=1}^{n} \lambda_{j} e^{-i a_{j} y} \equiv 0 .
$$

The functions $f_{i}(y)=e^{i a_{i} y}$ are linearly independent for distinct $a_{i}$, and analytic, so that $f(y)=\sum_{j=1}^{n} \lambda_{j} e^{-i a_{j} y}$ has isolated zeroes. Based on equation (6.20) we conclude that $\hat{c}(y)=0$ in $L^{2}(\mathbb{R})$, contradicting Lemma 4.2. Therefore $M$ must be invertible. This inversion procedure thus always produces a unique estimate of $S$ if the measurement locations are distinct.

We can also "solve" the inverse problem by choosing the unique function $S$ which satisfies equations $(6.17)$ and has minimal norm in a weighted $L^{2}$ space $L_{\delta}^{2}(\mathbb{R})$ with norm defined by the inner product

$$
<f, g>_{\delta}=\int_{-\infty}^{\infty} f\left(x_{1}\right) \bar{g}\left(x_{1}\right) \frac{1}{\delta\left(x_{1}\right)} d x_{1}
$$


where $\delta\left(x_{1}\right)$ is some real-valued non-negative function on $\mathbb{R}$. In this case, we have

$$
\int_{-\infty}^{\infty} S\left(x_{1}\right) c_{i}\left(x_{1}\right) d x_{1}=\int_{-\infty}^{\infty} S\left(x_{1}\right) c_{i}\left(x_{1}\right) \delta\left(x_{1}\right) \frac{1}{\delta\left(x_{1}\right)} d x_{1}
$$

where we must assume that $S=0$ wherever $\delta=0$. Thus the integral is understood to be taken only over that set where $\delta$ is non-zero. Equations (6.17) now take the form

$$
<S, c_{i} \delta>_{\delta}=d_{i}
$$

and the minimal norm solution is of the form

$$
S\left(x_{1}\right)=\delta\left(x_{1}\right) \sum_{i=1}^{n} \lambda_{i} c_{i}\left(x_{1}\right) .
$$

The idea is to choose $\delta\left(x_{1}\right)$ to have the same general form as $S\left(x_{1}\right)$, and so incorporate a priori information into the reconstruction based on (6.22) by forcing it to have the same general form. For example, if we know that $S$ is supported in the interval $[-b, b]$ we can choose $\delta(x) \equiv 1$ on $[-b, b]$ and $\delta(x) \equiv 0$ elsewhere. The optimal estimate of $S$ becomes

$$
S(x)=\chi_{[-b, b]} \sum_{i=1}^{n} \lambda_{i} c_{i}(x)
$$

where $\chi_{[-b, b]}$ is the characteristic function of the interval $[-b, b]$ and where the $\lambda_{i}$ are found by solving

for $j=1$ to $n$.

$$
\sum_{i=1}^{n}\left(\int_{-b}^{b} c_{i}(x) \bar{c}_{j}(x) d x\right) \lambda_{i}=d_{j}
$$

Whether we use a priori information or choose a uniform weighting on $L^{2}$, the reconstruction procedure is as follows: Compute the matrix $M$ defined by equation (6.19) and "measure" the data $d_{i}$ at the corresponding points $x_{1}=a_{i}$ on the top surface. Solve the system $M \lambda=d$ to obtain $\lambda_{1}, \ldots, \lambda_{n}$ and then compute an estimate of $S\left(x_{1}\right)$ from equation (6.18) or $(6.22)$. The stability of the finite data inversion is thus determined by the nature of the matrix $M$, and specifically, of its inverse. We can quantify the stability of the finite data inverse problem by studying the conditioning of the inverse of $M$ in various situations. This is done in the following section by studying the singular values of the matrix $M$. 


\section{Numerical Experiments}

We will now examine the finite data version of the inverse problem by using the previously described inversion procedure. In this section we apply the procedure to simulated data sets, both with and without noise. Our main focus is to examine the stability and resolution of back surface estimates with respect to various experimental parameters, specifically, the frequency of the input heat flux and the distribution of the measurement locations along the top surface of the sample. We also demonstrate how a priori assumptions about the nature of the corrosion can be incorporated into the inversion procedure, and the effects such assumptions have on stability and resolution.

There are a few points worth mentioning before we present the numerical results. The estimates of $S$ constructed using equation (6.18) lie in $H^{\infty}(\mathbb{R})$ and so the graph $x_{2}=S\left(x_{1}\right)$ would make sense as a curve in $\mathbb{R}^{2}$, except that the estimate will usually be complex-valued. This is not surprising, for a complex-valued $S$ makes perfect sense in the linearized version of the problem on which the inversion procedure is based. Estimates of $S$, especially in the presence of noise or the linearization error, will almost certainly have non-zero imaginary part. However, for those $S$ of "small" norm the linearized problem accurately reflects the full non-linear (with respect to $S$ ) direct problem and so the estimate of $S$ should be "mostly real", that is, it should have a relatively small imaginary component. This is indeed the case. A physically meaningful estimate of the true back surface $x_{2}=S\left(x_{1}\right)$ can be provided by either dropping the imaginary component or taking the modulus of the estimate. We choose the latter.

In the examples that follow we generate simulated test data using the full direct problem (2.2) with heating $g(x) \equiv 1$. The direct problem is solved by converting it into a boundary integral equation which is then solved numerically. The boundary integral formulation leads to a second kind Fredholm equation (explained below). The fact that $g$ is not compactly

supported nor even $L^{2}$ presents a minor problem. This can be fixed by simply subtracting off the function $u_{0}$ satisfying the direct problem with $S \equiv 0$. Recall that $u_{0}$ can be found explicitly by solving equation (3.5). The function $v=u-u_{0}$ then satisfies the boundary value problem 


$$
\begin{aligned}
\Delta v-i \omega v & =0 \text { in } \Omega \\
\frac{\partial v}{\partial \nu} & =0 \text { on } x_{2}=1 \\
\frac{\partial v}{\partial \nu} & =-\frac{\partial u_{0}}{\partial \nu} \text { on } x_{2}=S\left(x_{1}\right)
\end{aligned}
$$

If $S\left(x_{1}\right)$ is sufficiently rapidly decreasing then the Neumann data in the above boundary value problem is $L^{2}$ and the integral equation formulation becomes

$$
-\frac{1}{2} v(x)+\int_{\partial \Omega_{0}} \frac{\partial G}{\partial \nu_{y}}(x, y) v(y) d \sigma_{y}=-\int_{\partial \Omega_{0}} G(x, y) \frac{\partial u_{0}}{\partial \nu} d \sigma_{y}
$$

where $d \sigma_{y}$ is surface measure on $\partial \Omega_{0}$ and $G(x, y)=\Gamma(|x-y|)$. The integral equation is solved using Nyström's method (see [2]) with appropriate quadrature rules on the interval $(-\infty, \infty)$ that arises when the boundary of $\Omega_{0}$ is parameterized. The boundary integral approach is exceptionally fast and accurate and provides estimates of the solution only on the boundary of $\Omega_{0}$, the only place that we need the solution.

To illustrate the general procedure and to show that the inversion algorithm provides reasonable estimates, we begin with a simple example. We apply the inversion procedure to data generated using the back surface

$$
S(x)=\frac{e^{-(x-1)^{2}}}{10}+\frac{e^{-(x+2)^{2} / 2}}{5} .
$$

We use a heating frequency of $\omega=1$. As a first step the function $c(x)$ defined in Lemma 4.1 is computed by solving the system (4.10) with $a=0$ numerically and then computing $\phi_{0}=-2 \Gamma+v_{0}$, again via Nyström's method. These quantities are precomputed and stored rather than re-computed every time they are needed, for the function $c(x)$ depends only on $\Omega_{0}$, not $S$. The same is true of the matrix $M$. The entries of the matrix are computed by using equation (6.19) and an appropriate quadrature rule on $(-\infty, \infty)$. In the example below the data is computed using the full direct problem. The temperature data vector $d$ is computed at 21 equally spaced points on the top surface, $x_{1}=a_{i}$ where $a_{i}=-5+\frac{i}{2}$ for $i=0$ to 20 . We then invert the $21 \times 21$ system $M \lambda=d$ to find $\lambda$ and return an estimate of $S$ via equation (6.18). The estimate of $S$ is computed at a suitable number of points on the range of interest, in this case from -5 to 5 . The reconstruction is shown in Figure 2. The dotted line is the actual function $S(x)$ and the solid line is the reconstructed version. 


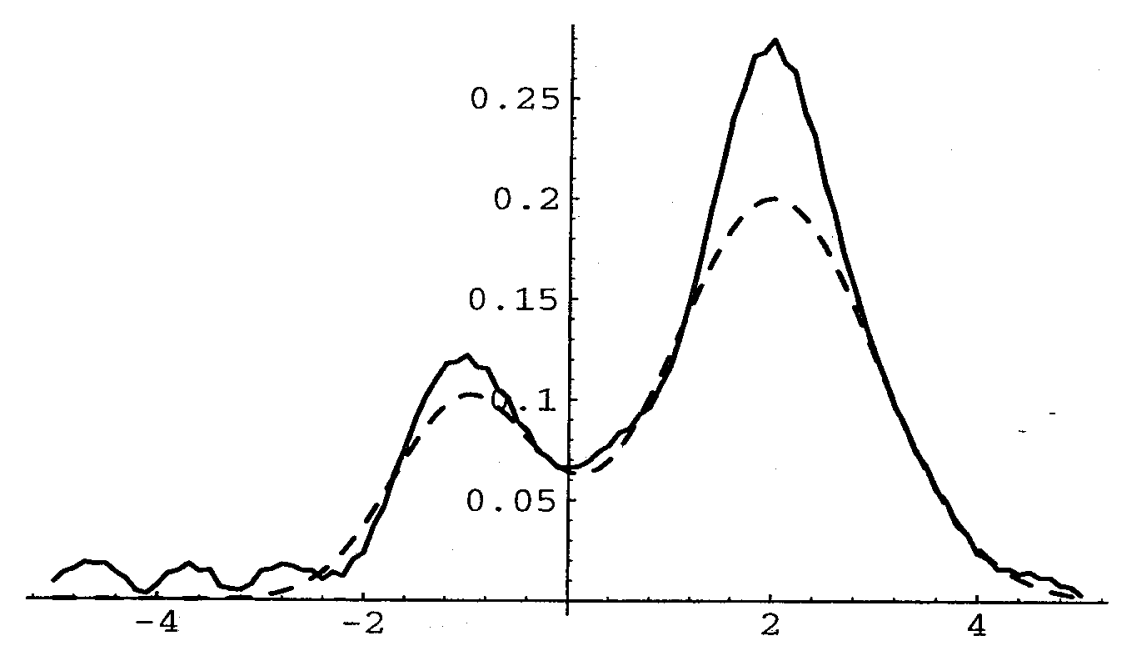

Figure 2: Reconstruction of $S(x)=\frac{e^{-(x+1)^{2}}}{10}+\frac{e^{-(x-2)^{2} / 2}}{5}$.

\section{Stability}

Of particular interest is the sensitivity of the inversion procedure with respect to various experimental parameters, e.g., heating frequency and measurement locations. The first task is to quantify the stability or conditioning of the finite data inverse problem. One sensible way to do this is to perform a singular value decomposition on the matrix $M$ defined by equation (6.19) and examine the magnitude of the singular values. When the singular values are small the inversion of $M \lambda=d$ magnifies small perturbations in $d$. Put another way, small singular values mean that relatively large changes in $S$ (and so in $\lambda$ ) produce relatively small changes in the data, so that perturbations in the back surface are "hard to see." Our goal in choosing experimental parameters is therefore to make the singular values of $M$ as large as possible, within certain limits. We should remark that one can define the condition number of $M$ as the ratio of the largest to smallest singular values and then attempt to quantify stability using this single number. This is not always good approach in the present setting, as later examples will show.

Let us begin by examining how the stability of the inversion procedure depends on the locations of the temperature measurements on the top surface. In the following examples we fix the heating frequency at $\omega=1$ and take measurements of the resulting temperature at 21 equally spaced locations on the interval $[-a, a]$ for several values of $a$. The resulting measurement locations are therefore of the form $a_{i}=-a+\frac{a \times i}{10}$ for $i=0, \ldots, 20$. In each case the matrix $M$ is computed and a singular value decomposition is performed. Let the 


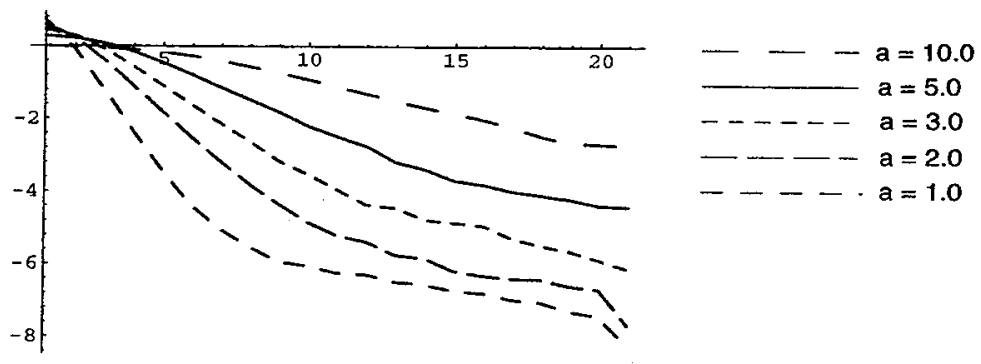

Figure 3: $\log _{10}\left|\alpha_{i}\right|$ versus $i$ for various values of $\bar{a}$.

singular values of $M$ be denoted by $\alpha_{i}, i=1$ to 21 , arranged in descending order. In Figure 3 we plot the quantity $\log _{10}\left|\alpha_{i}\right|$ versus $i$ for the cases $a=1,2,3,5,10$.

It is apparent that as the measurement locations become more spread out (as $a$ gets larger) the singular values decay more slowly and hence the inversion procedure becomes more stable. In light of Theorem 5.2 this is not surprising. When the measurement locations are close together we are able to resolve higher spatial frequencies in the data and so we are able to estimate higher frequencies in the Fourier decomposition of $S$. But according to Theorem 5.2, these are exactly the portions of $S$ that are difficult to reconstruct-they are heavily damped out in the data. The finite data version of the problem reflects this, with a full 6 orders of magnitude variation for the smallest singular values between the cases $a=1$ and $a=10$.

Another way to look at the stability of the various experimental configurations is to suppose that we have an "error magnification tolerance" $E$, and that in the inversion procedure we disregard all singular vectors whose singular values are less than $\frac{1}{E}$. This idea has been used in studying the stability for the impedance imaging problem [8]. The inversion procedure is then stabilized at the expense of rendering those components of $S$ lying in the span of the corresponding functions invisible. Figure 4 shows the number of singular values of $M$ which satisfy $\alpha_{k}>\frac{1}{E}$ versus $\log _{10}(E)$ for $E$ from 1 to $10^{-9}$. As in the previous examples, the matrix $M$ is $21 \times 21$ and we use measurement locations on the top surface $a_{i}=-a+\frac{a \star i}{10}$, $i=0, \ldots, 20$ for $a=1,2,3,5,10$. The heating frequency is $\omega=1$. 


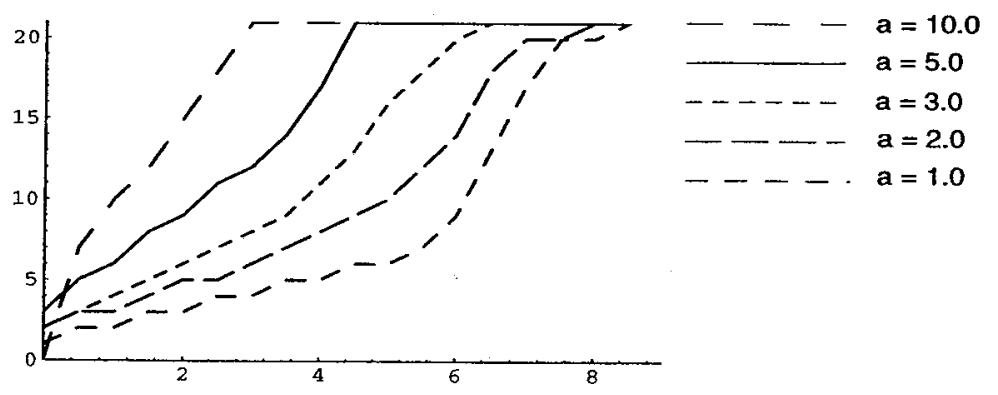

Figure 4: Number of singular values with $\alpha_{i}>\frac{1}{E}$ versus $\log _{10}(E)$ for various values of $a$.

Figure 4 also makes clear that as the measurement locations become spread out more singular values satisfy $\alpha_{i}>\frac{1}{E}$. The inversion procedure then admits more basis functions, presumably improving the fidelity of the reconstruction. In the two cases below we perform the actual reconstruction with $E=100$ (so only singular values greater than 0.01 are admissible) and add a small amount of random noise to the data (equal to 10 percent of the maximum signal strength). We then perform a reconstruction which omits all basis vectors whose corresponding singular values are less than $\frac{1}{E}$. Figure 5 illustrates the case in which the measurements locations are equally spaced from -5 to 5 ; there are 9 admissible singular values.

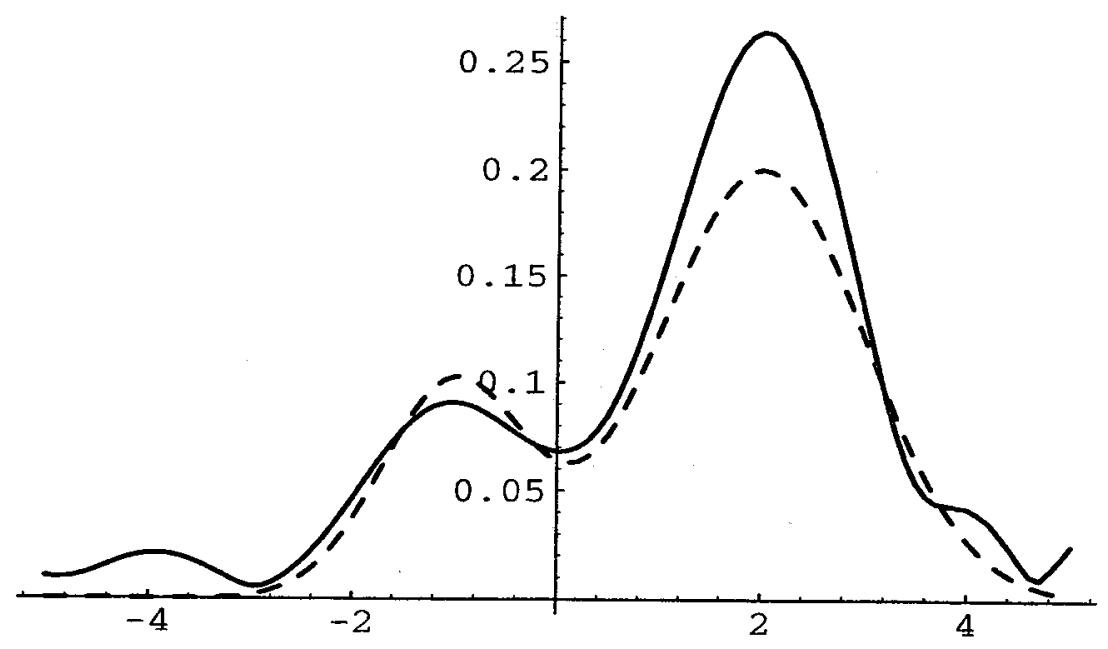

Figure 5: Reconstruction of $S(x)$ for 21 measurements on $[-5,5]$, tolerance $E=10^{2}$.

In Figure 6 we take the 21 measurements on the smaller interval $[-1,1]$, which yields only 3 admissible singular values. 


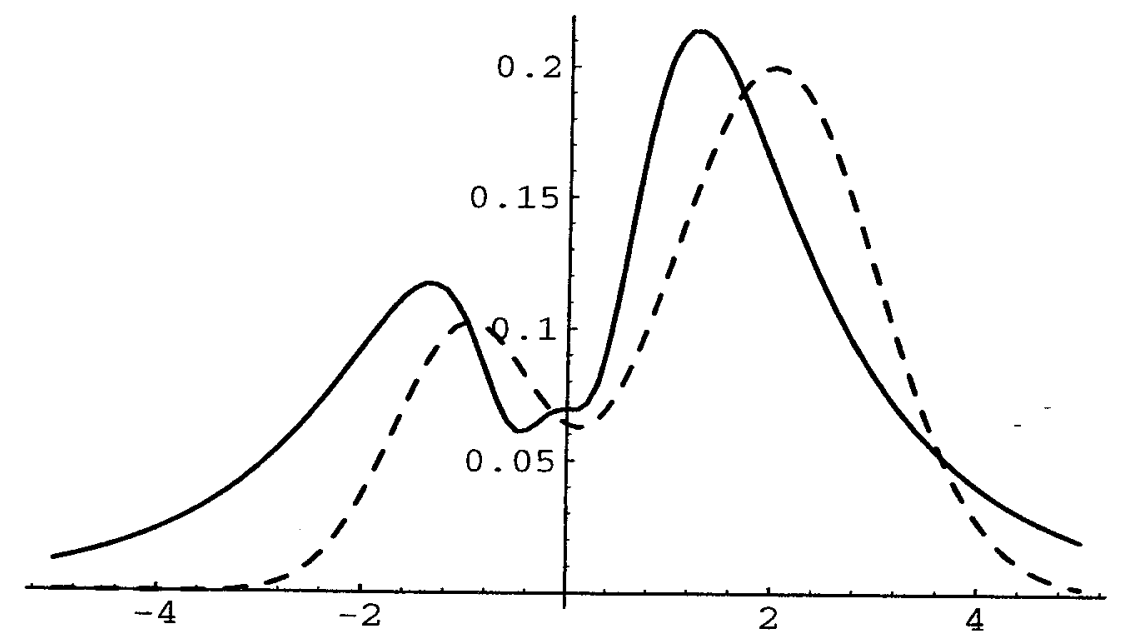

Figure 6: Reconstruction of $S(x)$ for 21 measurements on $[-1,1]$, tolerance $E=10^{2}$.

The reconstruction in Figure 6 is noticeably inferior to that of Figure 5 , but we have only 3 admissible basis functions with which to construct $S(x)$. Increasing the value of $E$ to admit more basis functions is not successful. Figure 7 illustrates what happens if we take $E=10^{4}$ with measurements on $[-1,1]$. Now 5 singular values are admissible, but the reconstruction is overwhelmed by noise.

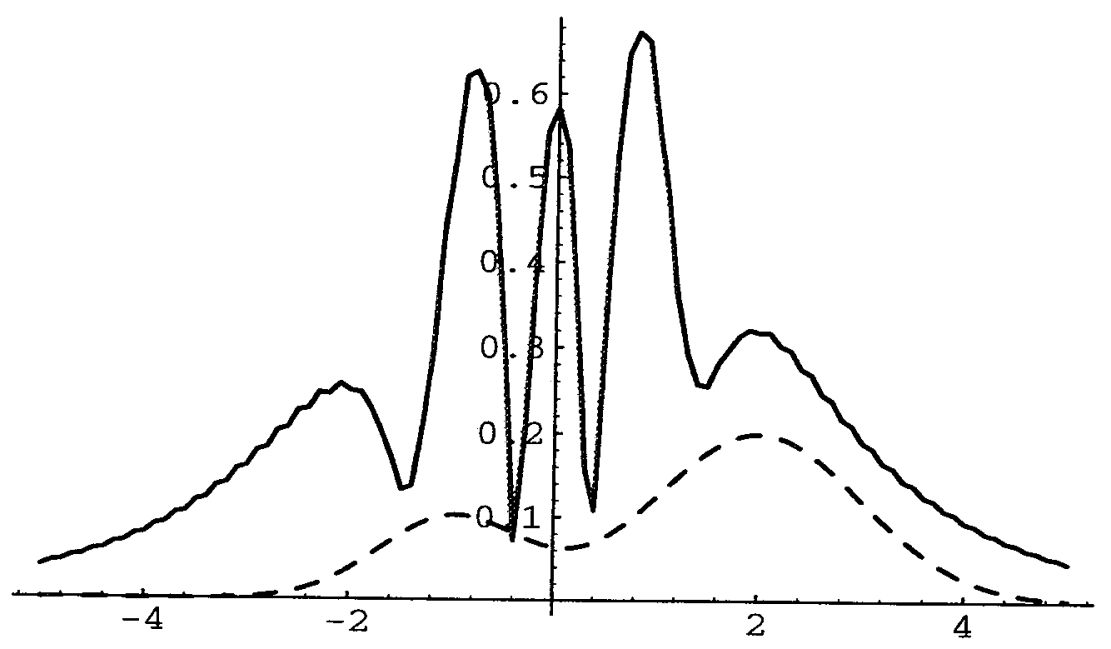

Figure 7: Reconstruction of $S(x)$ for 21 measurements on $[-1,1]$, tolerance $E=10^{4}$.

The moral seems clear: for maximum stability with a fixed number of measurement locations, we should spread the measurements over as large a region as possible. There are limits to this approach, however. If we spread out the measurements we do gain stability, but we will no longer be able to estimate high frequencies in the Fourier decomposition of 
$S$. This is illustrated by Figure 8, where we take 21 noise-free measurements on the interval $[-10,10]$ and estimate $S$ with error tolerance $E=10^{2}$. In this case all of the singular values are admissible.

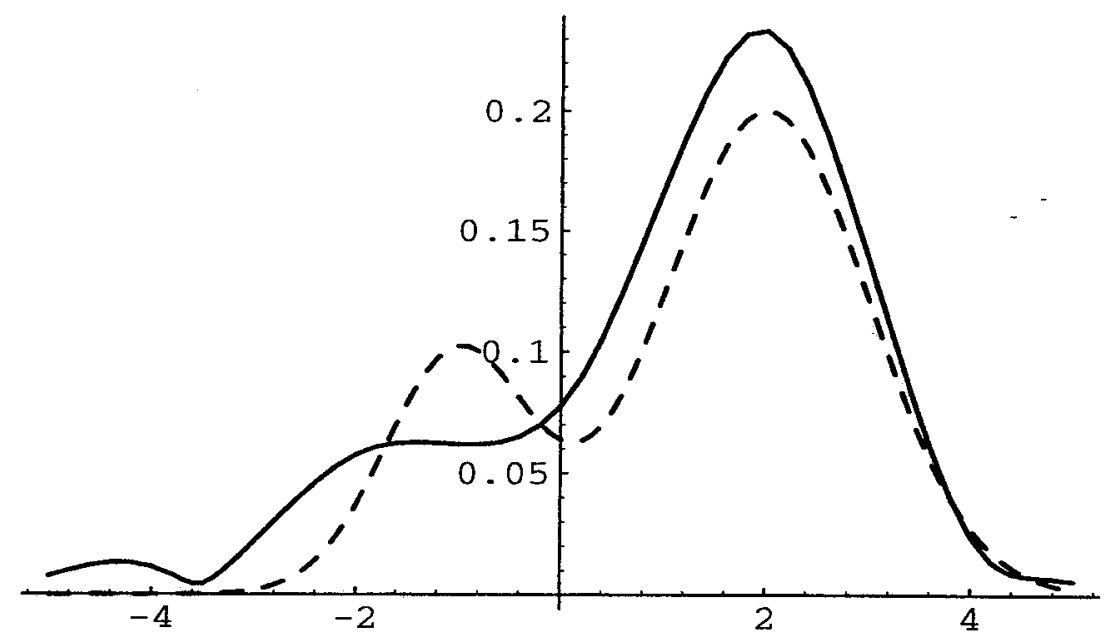

Figure 8: Reconstruction of $S(x)$ for 21 measurements on $[-10,10]$, tolerance $E=10^{2}$.

Despite the fact that the inversion is quite stable, our inability to resolve high frequencies results in a loss of resolution of small-scale detail in the reconstruction. With regard to the distribution of the measurement locations, the reconstruction process involves a compromise between stability and resolution of small-scale features.

In the next series of examples we examine the dependence of the stability on $\omega$, the frequency of the applied heat flux. We consider the cases $\omega=0.01,0.1,1.0,10.0,100.0$. In each case we take 21 equally spaced temperature measurements on the interval $[-5,5]$. Figure 9 shows $\log _{10}\left|\alpha_{i}\right|$ versus $i$ for each case.

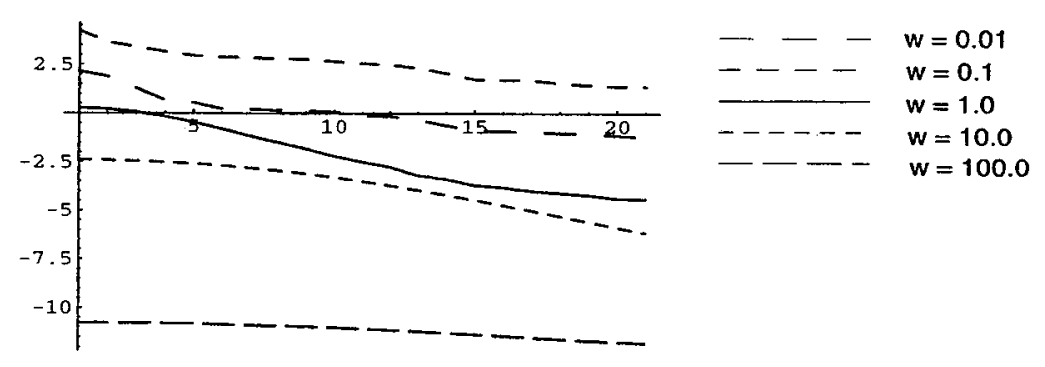

Figure 9: $\log _{10}\left|\alpha_{i}\right|$ versus $i$ for various values of $\omega$.

The figure illustrates that higher frequencies give rise to much smaller singular values. As before, it is instructive to consider the case in which we have an error tolerance $E$ and in 
the inversion process we omit those singular vectors whose singular values are less than $\frac{1}{E}$. Figure 10 shows the number of singular values which exceed $\frac{1}{E}$ for $E$ from $10^{-8}$ to $10^{15}$, for each of the frequencies.

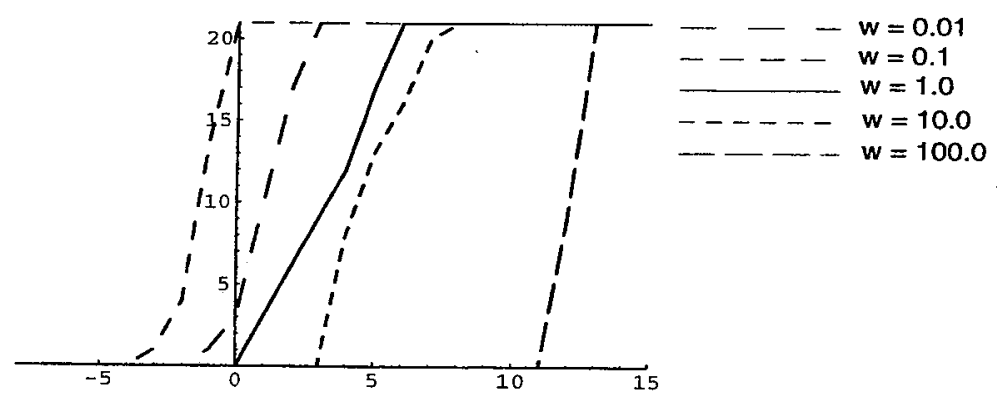

Figure 10: Number of singular values with $\alpha_{i}>\frac{1}{E}$ versus $\log _{10}(E)$ for various values of $\omega$.

Figure 10 clearly illustrates the situation. For $E=10^{-2}$ as before, $\omega=10$ and $\omega=100$ have no admissible singular values at all. The reconstruction (if carried out) is identically zero. The smaller singular values at higher frequencies are due to the fact that at higher frequencies the periodic heating penetrates very little into the sample and becomes more of a "skin effect." As a result very little energy reaches the back surface and even less returns to be measured on the top surface; at high frequencies the map taking $S$ into $d$ is essentially multiplication by zero. It is interesting to note that while higher frequencies produce smaller singular values, the condition number of $M$ for $\omega=100$ is only 10.3 , while the condition number for $\omega=0.01$ is 706.1 . Clearly, though, it's not enough to make the condition number small. The singular values themselves must be large enough for the inversion procedure to be stable in the presence of a fixed noise level.

While lower frequencies make the inversion process more stable, there are limits to how small we can make $\omega$ and maintain resolution. Figure 11 illustrates a reconstruction based on $\omega=0.1$. The parameters are otherwise identical to those that were used to produce Figure 5. All of the singular values are admissible. In fact, the smallest singular value is 0.063. As with the case in which the measurement locations were spread out over $[-10,10]$, we lose resolution at low temporal frequencies for the input flux. 


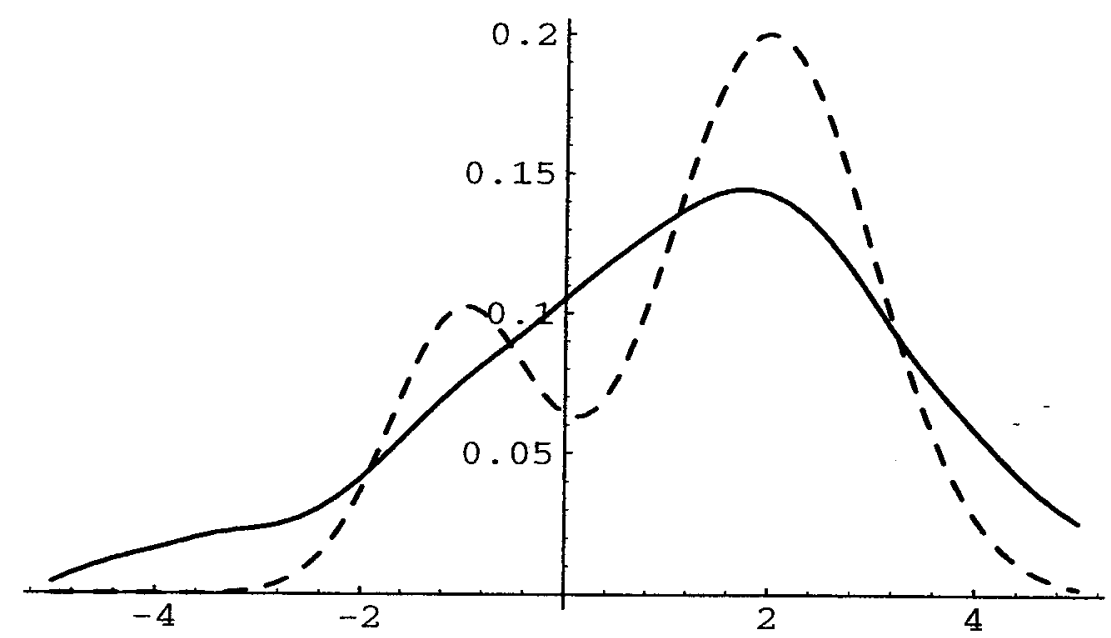

Figure 11: Reconstruction of $S(x)$ for 21 measurements on $[-5,5], \omega=0.1$, tolerance

$$
E=10^{2} .
$$

\section{Incorporating A Priori Information}

The preceding examples illustrate that the inversion procedure involves a compromise between stability and resolution. If the data points are too closely spaced, the inversion procedure is unstable. If the data points are too spread out, the inversion procedure becomes stable, but resolution is lost; measurements taken far from the support of the defect contain little information, because the heat diffuses very rapidly. Variations in the input heating frequency give rise to a similar phenomena. How shall we find the "best" experimental parameters? One useful possibility is to incorporate a priori information or assumptions into the inversion procedure. We will illustrate the idea by examining the problem under the assumption that the defect or function $S$ is supported in a known interval.

In the following examples we assume that the defect being imaged is supported in the interval $[-2,2]$. The only modification to the inversion procedure is that the matrix $M$ is computed in accordance with equation (6.21) and the function $S$ is estimated using equation (6.22). We will study the stability of the inversion procedure with respect to the distribution of the measurement locations on the top surface.

As in the previous cases, we choose measurement locations at $x_{1}=a_{i}$ on the sample top surface, where $a_{i}=-a+\frac{i}{10} a$ for $i=0$ to 20 . The heating frequency in all cases that follow 


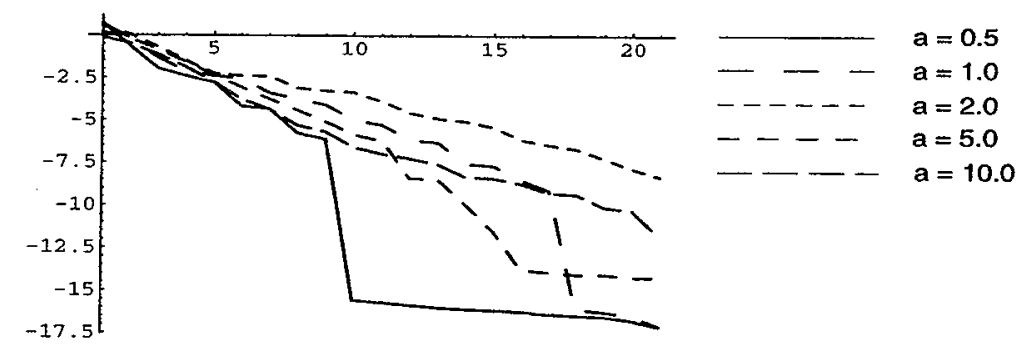

Figure 12: Singular values $\alpha_{i}$ versus $i$ for various values of $a$.

is $\omega=1$. Let us begin by examining the singular values of the inversion matrix $M$ for a few choices of $a$. In Figure 12 we plot the quantity $\log _{10}\left|\alpha_{i}\right|$ versus $i$ for $a=0.5,1.0,2.0,5.0,10.0$.

The figure shows that the best conditioning for the inverse problem occurs at $a=2$, when the measurement locations are distributed approximately in the same interval in which the defect is assumed to be supported. As before, closely spaced locations give rise to an ill-conditioned problem. However unlike the previous cases widely spaced nodes also result in poor conditioning. When $M$ is computed using equation (6.21) those rows of $M$ corresponding to measurement locations far from the support of $S$ are very nearly set to zero since the function $c\left(x-a_{i}\right)$ is rapidly decreasing away from $a_{i}$.

If an error magnification tolerance $E$ is specified, we can plot the number of allowable singular values $\alpha_{i}>\frac{1}{E}$ versus $\log _{10}(E)$ for the different node spacings:

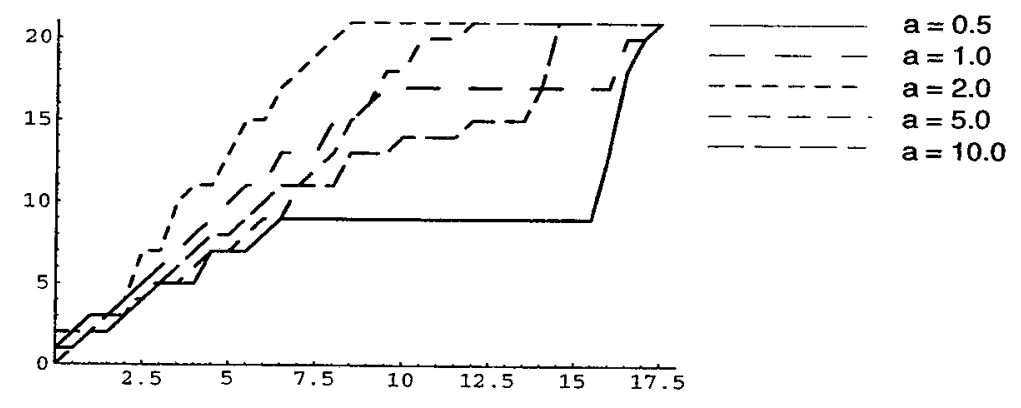

Figure 13: Number of singular values with $\alpha_{i}>\frac{1}{E}$ versus $\log _{10}(E)$ for various values of a.

As expected, $a=2.0$ allows more singular values for a fixed value of $E$ than any other choice for measurement spacing. It is useful to look at a few reconstructions based on this strategy. In the two cases below we take $E=300$ (so only singular values greater than $\frac{1}{300}$ are admissible) and add a small amount of random noise to the data (equal to 1 percent of 
the maximum signal strength). We then perform a reconstruction which omits all singular values less than $\frac{1}{E}$. The function defining the back surface is $S(x)=\frac{1}{10} e^{-2(x+1)^{2}}+\frac{1}{5} e^{-3(x-1)^{2}}$. Figure 14 illustrates the first case using $a=2$, the best choice according to Figure 13 . In this case 7 singular values are admissible.

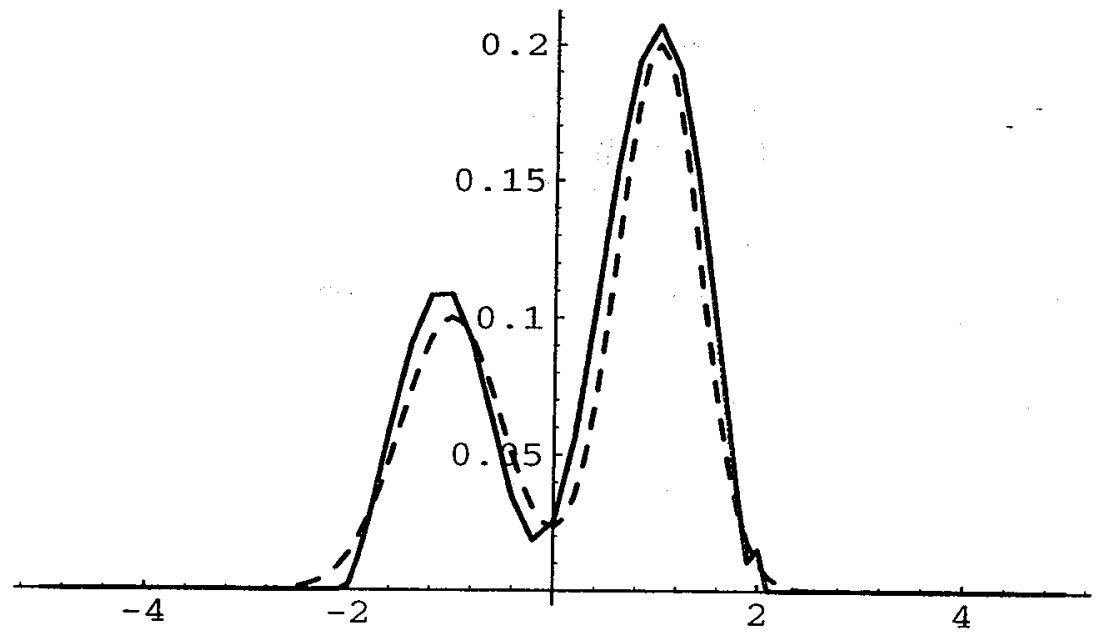

Figure 14: Reconstruction of $S(x)$ for 21 measurements on $[-2,2]$, tolerance $E=300$.

For $a=10$ we have 4 admissible singular values and the reconstruction shown in Figure 15 .

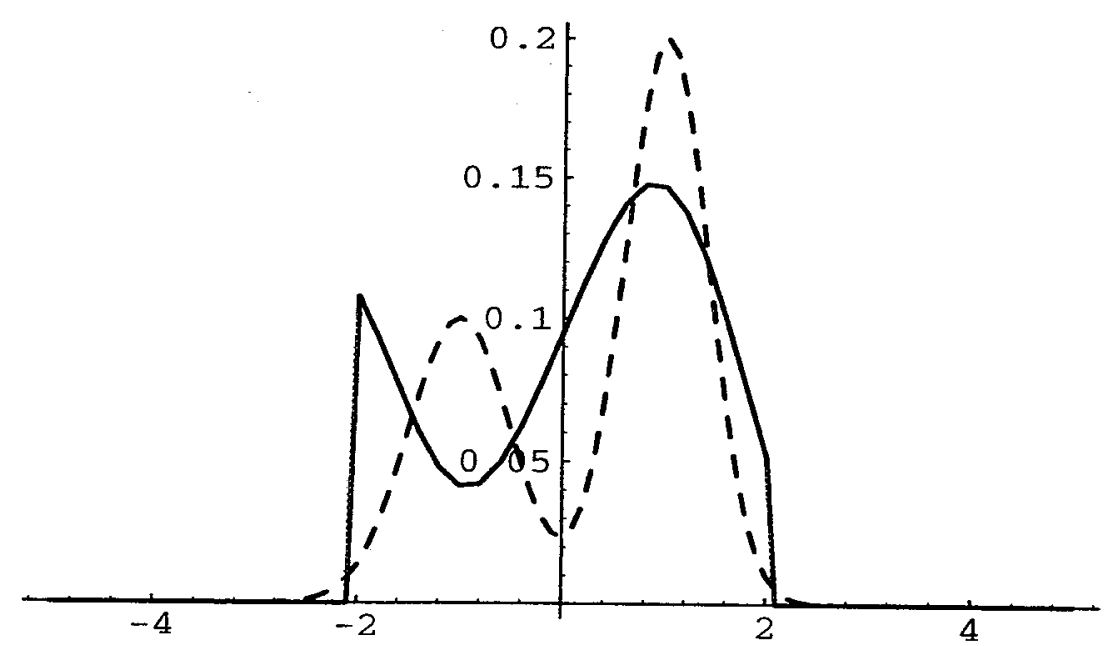

Figure 15: Reconstruction of $S(x)$ for 21 measurements on $[-10,10]$, tolerance $E=300$.

The case $a=0.5$ also yields 4 admissible singular values and the reconstruction shown in Figure 16. 


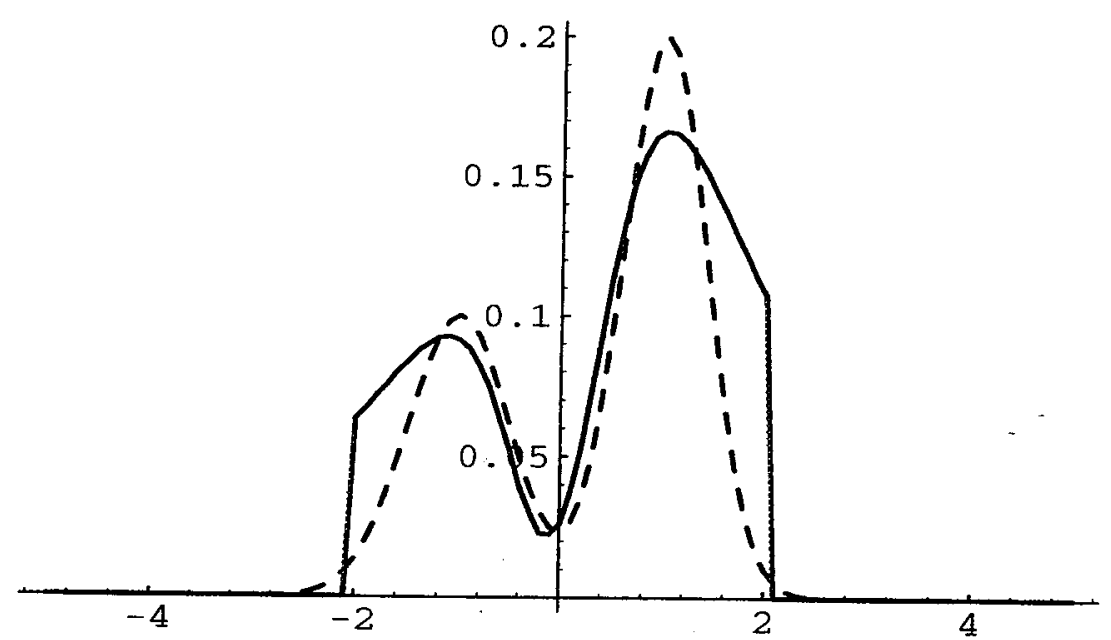

Figure 16: Reconstruction of $S(x)$ for 21 measurements on $[-0.5,0.5]$, tolerance $E=300$.

The actual reconstructions confirm that $a=2$ yields the most desirable results. Choosing $a$ significantly smaller or larger than the support of $S$ results in decreased stability and/or accuracy for the reconstruction.

Of course, the assumption that $S$ is supported in a given interval should be detrimental to the reconstruction if that assumption turns out to be false. In the following case we let $S(x)=\frac{1}{10} e^{-2(x+1)^{2}}+\frac{1}{5} e^{-3(x-4)^{2}}$ and perform the reconstruction under the assumption that $S$ is supported in the interval $[-2,2]$. We take measurements at 21 equally spaced location between -2 and 2 , the best case from above, and use an error tolerance $E=300$. The result is

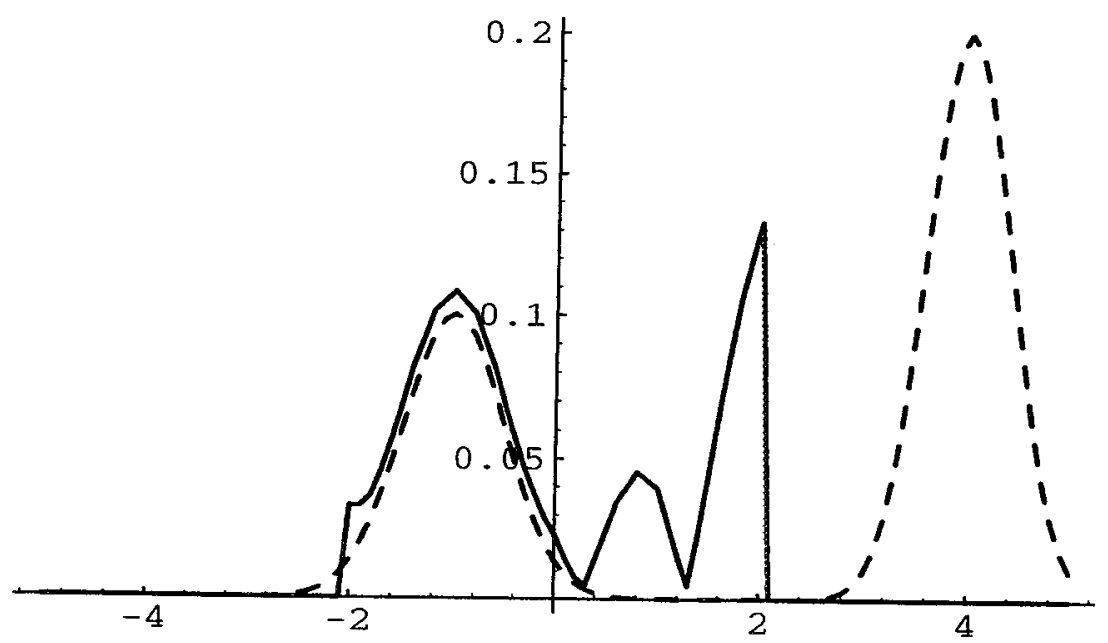

Figure 17: Reconstruction of $S(x)$ for 21 measurements on $[-2,2]$, tolerance $E=300$. 
The incorrect assumption obviously introduces errors into the reconstruction, although that portion of $S$ which is non-zero in the interval $[-2,2]$ is still recovered with reasonable accuracy.

\section{Concluding Remarks.}

In this paper we have investigated the inverse problem of recovering an unknown boundary portion of some object by applying a heat flux to an accessible portion of the boundary and measuring the resulting temperature response. We have considered a linearized version of the problem and found that the continuous version of the inverse problem, in which one has data at every point on the accessible portion of the surface, is extremely ill-posed. Indeed, the linearized version requires one to solve a first kind convolution integral equation for the unknown surface. The convolution kernel has a Fourier transform which dies rapidly at infinity, and so the inversion is extremely sensitive to the data at high spatial frequencies. We performed a variety of numerical studies which show that the ill-posedness is directly reflected in the finite data version of the problem, by the rapid decay of the singular values of the matrix which governs the inversion process. This ill-posedness depends on a number of factors; in particular, the locations of the measurements have a large effect on the conditioning of the inverse problem, and these effects mirror the behavior of the continuous version. We have also considered the effect of including a priori assumptions in the finite data inversion procedure, by weighting appropriate Hilbert spaces in which the solution $S$ resides. The inclusion of this information can help in determining the optimal locations for measurements on the top surface.

There are a number of interesting directions we could take from here. In our studies we used only the input flux whose magnitude is identically one on the top surface. Similar results can be obtained for more general fluxes, and this would allow one to study the effect. that the input heat flux has on sensitivity and resolution. The fully time-dependent case would also be of interest. The procedure presented in this paper would also work for a full three- dimensional problem, although qualitatively the results should be the same-the high spatial frequencies in the back surface should be difficult to see.

As mentioned earlier, the inversion process which chooses that function with minimal $L^{2}$ 
norm which is consistent with the measured data seems to act like a form of regularization for the inverse problem. It would be interesting to examine in what sense this is true, and how it relates to more traditional forms of regularization. It is also possible (and not difficult) to carry out the same minimization process in higher Sobolev spaces, e.g., $H^{1}$, and thus put a higher "penalty" on functions with oscillations. This too would make an interesting study. We would also like to examine conditions under which our inversion procedure is guaranteed to converge to the solution of the linearized inverse problem. 


\section{References}

[1] Abramowitz, Milton and Irene Stegun, Handbook of mathematical functions, Applied Mathematics Series, vol. 55. Washington: National Bureau of Standards, 1964. Reprinted 1968 by Dover Publications, New York.

[2] Atkinson, K.E., A survey of numerical methods for the solution -of fredholm integral equations of the second kind, SIAM, Philadelphia, PA, 1976.

[3] Banks, H.T. and F. Kojima, "Boundary shape identification problems in twodimensional domains related to thermal testing of materials," Quart. Appl. Math., Vol. 47 (1989), pp. 273-293.

[4] Banks, H.T., F. Kojima and W.P. Winfree, "Boundary estimation problems arising in thermal tomography," Inverse Problems 6 (1990), pp. 897-922.

[5] Byrne, Charles L. and Raymond M. FitzGerald, Reconstruction from partial information with applications to tomography, SIAM J. Appl. Math. Vol. 42, no. 4, (1982), pp. 933940 .

[6] Gisser, D.G., D. Isaacson, and J.C. Newell, Electric current computed tomography and eigenvalues, SIAM J. Appl. Math, Vol 50, No. 6, (1990), pp. 1623-1634.

[7] D. Isaacson, and M. Cheney, Effects of measurement precision and finite numbers of electrodes on linear impedance imaging algorithms. 1990, preprint.

[8] Dobson D. and F. Santosa, Stability and resolution analysis of an inverse problem in electrical impedance tomography - dependence on the input current patterns. SIAM J. Appl. Math., to appear.

[9] Folland, Gerald B., Introduction to partial differential equations. Princeton, NJ: Princeton University Press, 1976.

[10] Patel, P.M., S. K. Lau and D.P. Almond, A review of image analysis techniques applied in transient thermographic nondestructive testing, Nondestructive Testing and Evaluation, Vol. 6 (1992), pp.343-364. 
[11] Tricome, F.G. Integral equations. New York: Interscience Publishers, 1957.

[12] Wing, G. Milton A primer on integral equations of the first kind: the problem of deconvolution and unfolding. SIAM, 1991. 


\section{REPORT DOCUMENTATION PAGE}

Form Approved

OMB No. 0704-0188

Public reporting burden for this collection of information is estimated to average 1 hour per response, including the time for reviewing instructions, searching existing data sources, gathering and maintaining the data needed, and completing and reviewing the collection of information. Send comments regarding this burden estimate or any other aspect of this Dastions for reducing this burden, to Washington Headquarters Services, Directorate for Information Operations and Reports, 1215 Jefferson

(0) Washington, DC 20503.

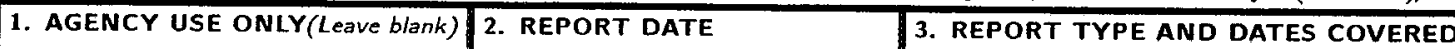

December 1994 Contractor Report

4. TITLE AND SUBTITLE AN INVERSE PROBLEM IN THERMAL IMAGING

. FUNDING NUMBERS

C NAS1-19480

WU 505-90-52-01

6. AUTHOR(S)

Kurt Bryan

Lester F. Caudill, Jr.

7. PERFORMING ORGANIZATION NAME(S) AND ADDRESS(ES)

Institute for Computer Applications in Science and Engineering

Mail Stop 132C, NASA Langley Research Center

Hampton, VA 23681-0001

9. SPONSORING/MONITORING AGENCY NAME(S) AND ADDRESS(ES)

National Aeronautics and Space Administration

Langley Research Center

Hampton, VA 23681-0001

8. PERFORMING ORGANIZATION REPORT NUMBER

ICASE Report No. 94-99

10. SPONSORING/MONITORING AGENCY REPORT NUMBER

NASA CR-195018

ICASE Report No. 94-99

\section{SUPPLEMENTARY NOTES}

Langley Technical Monitor: Michael F. Card

Final Report

Submitted to SIAM Applied Mathematics

12a. DISTRIBUTION/AVAILABILITY STATEMENT

12b. DISTRIBUTION CODE

Unclassified-Unlimited

Subject Category 64

13. ABSTRACT (Maximum 200 words)

This paper examines uniqueness and stability results for an inverse problem in thermal imaging. The goal is to identify an unknown boundary of an object by applying a heat flux and measuring the induced temperature on the boundary of the sample. The problem is studied both in the case in which one has data at every point on the boundary of the region and the case in which only finitely many measurements are available. An inversion procedure is developed and used to study the stability of the inverse problem for various experimental configurations.

\section{SUBJECT TERMS}

Inverse Problems; Dll-posed Problems; non-destructive testing; integral equations

15. NUMBER OF PAGES

34

16. PRICE CODE

20. LIMITATION

17. SECURITY CLASSIFICATION OF REPORT

Unclassified

NSN 7540-01-280-5500

18. SECURITY CLASSIFICATION
OF THIS PAGE
Unclassified

19

SECURITY CLAS
OF ABSTRACT OF ABSTRACT 